\title{
In Vitro Shoot Regeneration of Rice (Oryza sativa L.) Varieties under Artificial Drought Stress
}

\author{
Anindita Chakraborty*, Soumitra Chowdhury, Zahidul Haque, \\ Asim Kanti Dash and A. T. M. J. Mosnaz \\ Department of Genetic Engineering \& Biotechnology, \\ Shahjalal University of Science and Technology (SUST), Sylhet-3114, Bangladesh \\ *Corresponding author and Email: aninditamoury@yahoo.com
}

Received: 9 November $2017 \quad$ Accepted: 23 June 2018

\begin{abstract}
The shoot regeneration efficiency of two rice (Oryza sativa L.) varieties (Kasalath and BRRI dhan55) under artificial drought condition created by polyethylene glycol (PEG-6000) was investigated. Calli were induced in Murashige and Skoog (MS) medium supplemented with different concentrations ( 0 , $0.5,1.0,1.5,2.0,2.5,3.0$, and $4.0 \mathrm{mg} / \mathrm{L})$ of 2, 4-Dichlorophenoxyacetic acid (2, 4-D). Then, in vitro shoot regeneration under PEG (6000) was conducted on MS medium supplemented with $1.0 \mathrm{mg} / \mathrm{L}$ $\mathrm{NAA}+2.0 \mathrm{mg} / \mathrm{L} \mathrm{BAP}+1.0 \mathrm{mg} / \mathrm{L}$ kinetin that gave the highest number of shoots. Shoot regeneration in both varieties reduced with increased levels of PEG $(0.5,1.0,2.0,3.0$ and $4.0 \%)$. Regeneration efficiency of BRRI dhan55 in drought stress was higher compared to Kasalath. Thus, in vitro screening of plantlets under PEG can be used to achieve fast and reliable selection for tolerance against drought.
\end{abstract}

Keywords: Drought, polyethylene glycol, shoot regeneration.

\section{Introduction}

Drought is one of the major abiotic stresses causing severe yield loss in crop plants worldwide (Akte et al., 2016). Drought stress impairs physiochemical processes of plants such as photosynthesis, respiration, nutrient uptake and metabolism (Piwowarczyk et al., 2014). Plants respond to drought stress through morphological, physiological and metabolic adjustments through different mechanisms (Akte et al., 2016). However, this response often leads to accumulation of reactive oxygen species (ROS) such as superoxide, hydrogen peroxide $\left(\mathrm{H}_{2} \mathrm{O}_{2}\right)$, hydroxyl radicals and singlet oxygen. These ROS cause peroxidation of lipid membrane, membrane injuries, protein degradation and enzyme deactivation and lead to the ultimate death of plant (Phung et al., 2011; Akte et al., 2016).

Rice (Oryza sativa L.) is the most important cereal crop in the world (Akte et al., 2016). In Bangladesh, it is a staple food and is grown almost all over the country. However, its average yield is low (2.94 t/ha) compared to other rice growing countries such as China (6.8 t/ha) (BBS, 2012; FAOSTAT, 2014). The reasons could be adverse climatic conditions such as flood, salinity and drought (Akte et al., 2016). Rice is highly susceptible to drought stress and drought causes substantial yield reduction at the 
reproductive stage. It causes $50 \%$ loss of production of rice globally (Wani et al., 2010; Swamy et al., 2012). In the South and South East Asia, drought causes up to $40 \%$ yield loss (Pandey and Bhandari, 2006; IRRI, 2009). In Bangladesh, this loss ranges from $37-73 \%$ depending on the grain development stage (Mahmood, 2004). Thus, it is imperative to find rice varieties that will be adapted to these conditions.

Screening drought tolerant plants is a way to sustain production under drought environment. However, screening in field is laborious, time consuming and is dependent on resources such as worker, field and power. In vitro selection method could be used as it is simple, ideal and effective for screening large set of germplasms (Kulkarni and Deshpande, 2007; Kacem et al., 2017). Artificial drought stress can be achieved in vitro by addition of different osmatic agents such as mannitol, polyethylene glycol (PEG), sucrose or sorbitol in the medium for the culture of explants (Kacem et al., 2017). Among these, PEG is the most common and widely used chemical to study plant responses to drought stress (Wani et al., 2010). PEG is non-ionic water-soluble polymer having wider range of molecular weights (Wani et al., 2010; Tripathy, 2015). PEG (6000 MW) has been used in in vitro screening of plants such as rice (Wani et al., 2010; Akte et al., 2016; Sabesan and Saravanan, 2016), tomato (Kulkarni and Deshpande, 2007), wheat (Kacem et al., 2017), peanut (Meher et al., 2017) against drought stress. This is because PEG can induce water stress without creating any detrimental effect on the plant. It is also useful in modifying the osmotic potential of culture medium (Wani et al., 2010; Tripathy, 2015; Meher et al., 2017). The present study was conducted to determine the effect of artificial drought stress created by PEG (6000) on callus derived shoot regeneration of two drought tolerant varieties, BRRI dhan55 and Kasalath. The present study aimed at providing a basis for selecting the varieties for further drought tolerance breeding program.

\section{Materials and Methods}

\subsection{Plant material and experimental site}

Mature seeds of two drought tolerant rice varieties, BRRI dhan55 and Kasalath were used in the experiment. Seeds of BRRI dhan55 and Kasalath were collected from seed merchant and local farmers respectively. BRRI dhan55 was developed by Bangladesh Rice Research Institute (BRRI) and was released for commercial cultivation in 2011, while Kasalath is a landrace (Parvin et al., 2015). The experiment was conducted in the Plant Genetic Engineering Laboratory of the Department of Genetic Engineering and Biotechnology, Shahjalal University of Science \& Technology (SUST), Sylhet.

\subsection{Callus induction}

Manually dehusked seeds were sterilized by $70 \%$ alcohol for 1 minute and $1 \%$ sodium hypochlorite (Clotech) for 10 minutes with few drops of tween-20. To induce callus, the seeds were cultured on MS medium in test tubes. The medium was supplemented with different concentrations of 2 , 4-D $(0,0.5,1.0,1.5,2.0$, 2.5, 3.0, and $4.0 \mathrm{mg} / \mathrm{L}$ ). In each test tube, one seed was explanted and inoculation was done in a laminar air flow cabinet. The test tubes were incubated in a growth room at $25 \pm 3{ }^{\circ} \mathrm{C}$ for 16 hours of light $\left(200 \mu \mathrm{mol} \mathrm{m} \mathrm{m}^{-2} \mathrm{~s}^{-1}\right)$ per day. Three weeks after inoculation, the calli were removed from the seeds and were transferred into fresh nutrient medium. Callus induction frequency for the two varieties was recorded three weeks after inoculation. All calli from a single seed were considered as one. The percent frequency of callus induction was calculated as the number of calli obtained/ total number of seeds cultured multiplied by 100 .

\subsubsection{Shoot regeneration}

Shoot regeneration involved two steps. Firstly, there was optimization of the shoot regeneration media. Then, the best shoot regeneration medium was used to investigate the impact of drought stress on regeneration of the two rice varieties under PEG (6000). 
To optimize shoot regeneration from calli, onemonth old good-quality calli were excised and transferred into MS medium contained in test tubes supplemented with eight different combinations of NAA and two cytokinins (BAP and Kinetin) (Table 1).

To investigate the effect of drought stress on shoot regeneration of the two rice varieties, different concentrations of PEG $(0.5,1.0,2.0$, 3.0 and $4.0 \%$ ) were supplemented into the medium. All cultures were incubated in growth chambers similar to that mentioned earlier. The frequencies of plantlet regeneration were calculated on the basis of shoot initiation from the calli. The shoot regeneration frequency (number of plantlets/ total number of calli x 100) before and after PEG treatment was also recorded. The medium without PEG was considered as the control.

\section{Results and Discussion}

\subsection{Effect of 2, 4-D on Callus Induction}

Callus induction started from the scutellum of the seeds after 3 days of culture in both varieties.
It increased with increased concentration of 2, 4$\mathrm{D}$ then declined (Figure 1). Callus induction in rice variety Kasalath was the highest $(73 \%)$ in the medium supplemented with $2.0 \mathrm{mg} / \mathrm{L}$ of 2 , 4D (Figure 5-A) whereas for BRRI dhan55 maximum $(67 \%)$ induction was in $2.5 \mathrm{mg} / \mathrm{L}$ of 2 , 4-D.

These results are consistent with earlier reports. Shahsavari et al. (2010), Haque et al. (2013), Pawar et al. (2015) and Upadhyaya et al. (2015) found higher callus induction with $2.0 \mathrm{mg} / \mathrm{L}$ of 2 , 4-D while Hussain et al. (2010) and Chakraborty et al. (2017) showed maximum callus induction with 2-4 $\mathrm{mg} / \mathrm{L}$ of 2, 4-D. Callus induction frequency might have been influenced by genotypes. Except for 2.5 and $4.0 \mathrm{mg} / \mathrm{L}$ of 2, 4-D concentration, Kasalath had the highest callus induction than BRRI dhan55 in all 2, 4-D concentrations. On average, $47 \%$ of inoculated seeds developed callus in Kasalath compared to $39 \%$ in BRRI dhan55. Earlier studies had also revealed that callus induction in rice could be genotype specific (Shahsavari et al., 2010; Hussain et al., 2010; Upadhyaya et al., 2015).

Table 1. Shoot regeneration medium (MS medium containing $1.0 \mathrm{mg} / \mathrm{L}$ NAA as constant with different concentration of BAP and Kinetin)

\begin{tabular}{cc}
\hline Media Name & Type of combination \\
\hline K1 & BAP $1.0 \mathrm{mg} / \mathrm{L}+$ Kinetin $0.5 \mathrm{mg} / \mathrm{L}$ \\
K2 & BAP $1.0 \mathrm{mg} / \mathrm{L}+$ Kinetin $1.0 \mathrm{mg} / \mathrm{L}$ \\
K3 & BAP $1.0 \mathrm{mg} / \mathrm{L}+$ Kinetin $1.5 \mathrm{mg} / \mathrm{L}$ \\
K4 & BAP $1.0 \mathrm{mg} / \mathrm{L}+$ Kinetin $2.0 \mathrm{mg} / \mathrm{L}$ \\
K5 & BAP $2.0 \mathrm{mg} / \mathrm{L}+$ Kinetin $0.5 \mathrm{mg} / \mathrm{L}$ \\
K6 & BAP $2.0 \mathrm{mg} / \mathrm{L}+$ Kinetin $1.0 \mathrm{mg} / \mathrm{L}$ \\
K7 & BAP $2.0 \mathrm{mg} / \mathrm{L}+$ Kinetin $1.5 \mathrm{mg} / \mathrm{L}$ \\
K8 & BAP $2.0 \mathrm{mg} / \mathrm{L}+$ Kinetin $2.0 \mathrm{mg} / \mathrm{L}$ \\
\hline
\end{tabular}




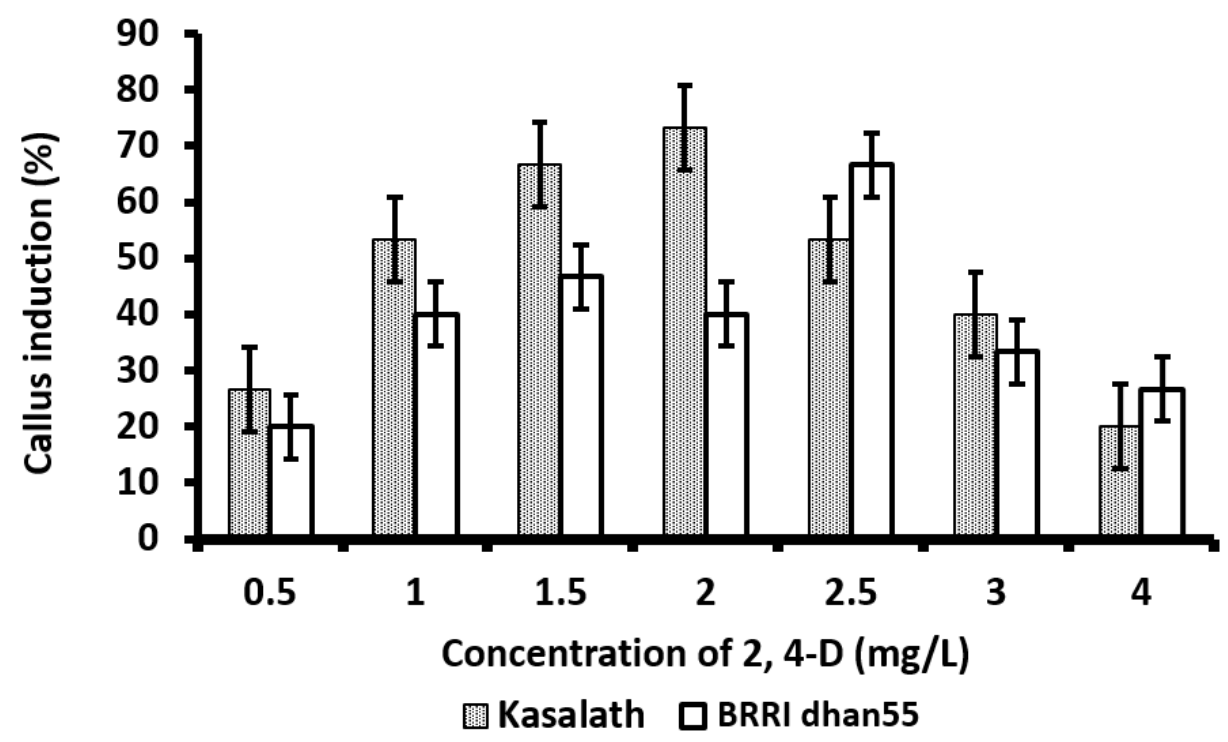

Figure 1. Callus induction of Kasalath and BRRI dhan55. Bars are standard error.

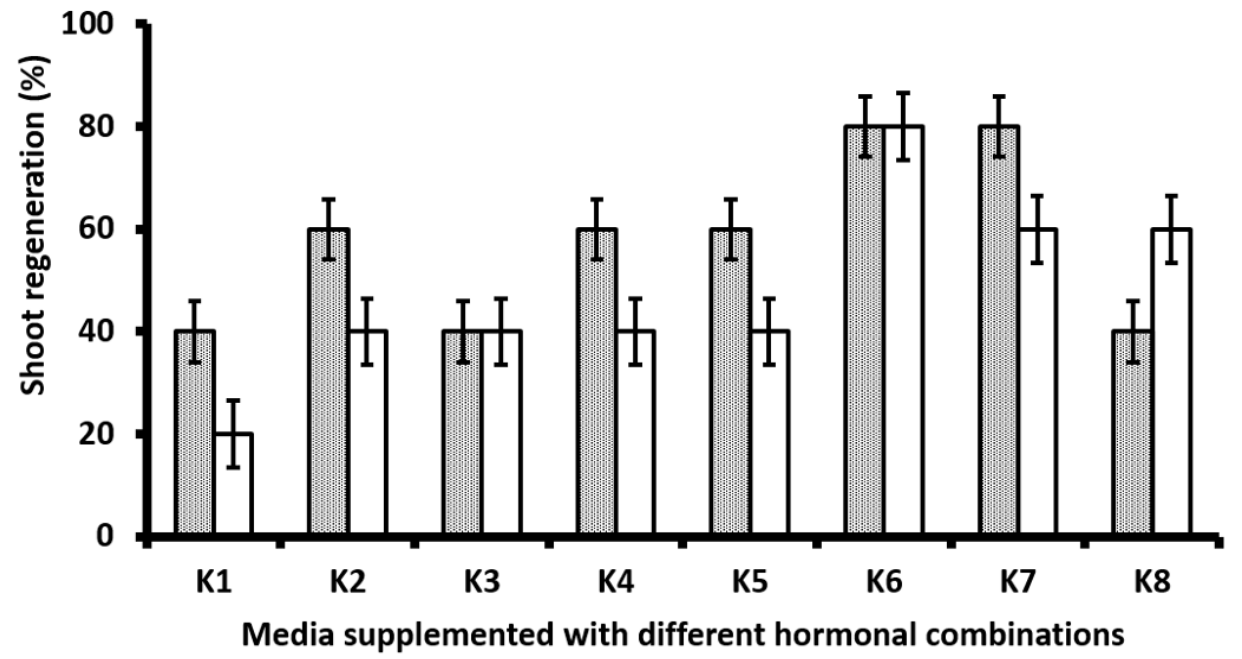

$\square$ Kasalath $\square$ BRRI dhan55

Figure 2. Effect of different hormonal combinations on shoot regeneration from calli. Bars are standard error. 
2, 4-D is a synthetic auxin that is commonly used as a plant growth regulator to produce callus. Many studies suggest that MS medium supplemented with just 2, 4-D can produce more calli (Shahsavari et al., 2010). Other studies, however, showed that it is the combination of 2 , 4-D and other plant growth regulators that induce more calli. But this is controversial. For instance, Shahsavari et al. (2010) did not find any significant effect of a combination of NAA or kinetin with 2, 4-D in callus induction. However, Mohd Din et al. (2016) found high level of callus induction in medium supplemented with 2, 4-D and combination of BAP or NAA. The present study also revealed that 2, 4-D inevitably controls callus initiation of rice. MS medium supplemented with 2, 4-D alone produced callus from both varieties (Kasalath and BRRI dhan55).

Further, callus initiation depended on the concentration of 2, 4-D in the medium. Less number of calli were initiated at concentration above a certain level. This is consistent with reports of Wani et al., (2011); Mohd Din et al., (2016) who also showed reduction of callus initiation with the increasing concentration of 2 , 4-D in rice. Suppression of calli initiation at high concentration of 2, 4-D could be due to the residual effect of 2, 4-D on re-differentiation in the mitotic stage (Mohd Din et al., 2016). This means an optimum concentration of 2, 4-D is desirable to get maximum number of rice callus.

\subsection{Optimization of auxin and cytokinin for shoot regeneration}

MS medium supplemented with different combinations of auxin and cytokinins were optimized for shoot regeneration system that could work for both varieties. About 4-5 week old embryogenic calli were selected and placed on MS medium (K1 to K8) supplemented with NAA $(1.0 \mathrm{mg} / \mathrm{L})$, BAP $(1.0$ or $2.0 \mathrm{mg} / \mathrm{L})$ and kinetin $(0.5,1,1.5$ or $2 \mathrm{mg} / \mathrm{L})$. Results showed a higher regeneration frequency of $80 \%$ for both varieties in K6 medium containing MS medium with $1.0 \mathrm{mg} / \mathrm{L} \mathrm{NAA}+2.0 \mathrm{mg} / \mathrm{L} \mathrm{BAP}+1.0 \mathrm{mg} / \mathrm{L}$ kinetin (Figure 2, Figure 5-B). But the regeneration capacity of Kasalath was higher than that of BRRI dhan55 in all media. These results are closely related to those of Shahsavari et al. (2010) who showed maximum regeneration frequency of upland rice cultivars on MS medium supplemented with $0.5 \mathrm{mg} / \mathrm{L} \mathrm{NAA}+2.0$ $\mathrm{mg} / \mathrm{L} \mathrm{BAP}+2.0 \mathrm{mg} / \mathrm{L}$ kinetin. Furthermore, they found that some rice cultivars had higher regeneration capacity. Hussain et al. (2010) also supplemented MS medium with NAA and BAP and found higher plantlet regeneration in MS medium and found higher plantlet regeneration in MS medium supplemented with either $1 \mathrm{mg} / \mathrm{L}$ $\mathrm{NAA}+2 \mathrm{mg} / \mathrm{L} \mathrm{BAP}$ or $1 \mathrm{mg} / \mathrm{L} \mathrm{NAA}+4 \mathrm{mg} / \mathrm{L}$ BAP based on varieties.

Hormonal balance between auxin and cytokinin has been showed to significantly alter in vitro callus, shoot and root formation. Auxins and cytokinins interact synergistically, antagonistically and additively to influence plant responses. But this is also based on type of tissue and plant species (Coenen and Lomax, 1998).

Thus, the ratio of auxin to cytokinin is vital in in vitro shoot formation. Previous studies indicate that plant regeneration in rice from callus has been achieved under different combinations of auxins to cytokinins (Hussain et al., 2010; Wani et al., 2011; Mohd Din et al., 2016). In the present study, the concentration of NAA, BAP and Kinetin in MS medium was crucial for the shoot regeneration and depended on variety too.

\subsection{Effects of PEG on shoot regeneration of rice}

Polyethylene glycol had a distinctive effect on shoot regeneration from callus. In both varieties, shoot regeneration declined with increased PEG concentration (Figure 3). In the regeneration medium, K6 with PEG concentration of 0.5 and $1.0 \%$, the frequency of shoot regeneration was higher (75 and $70 \%$, respectively) which was similar to the regeneration frequency in unstressed condition (80\% in K6 media, without PEG). The lowest percentage of shoot regeneration was obtained in both varieties under severe water stress created by $4 \%$ PEG. 


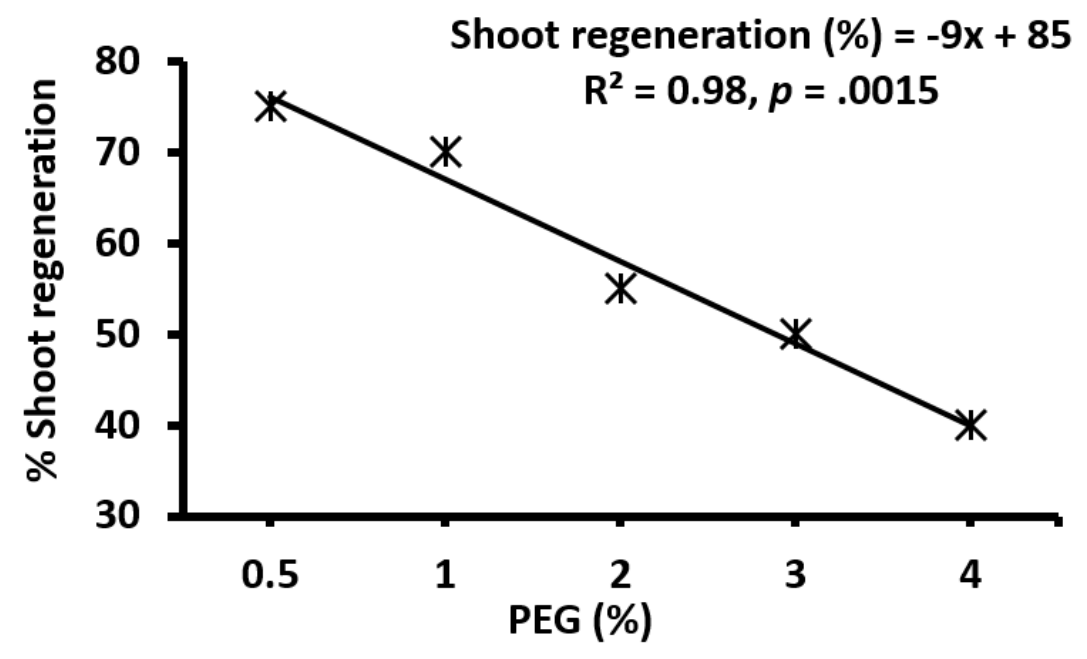

Figure 3. Effect of PEG supplement into MS medium on rice shoot regeneration.

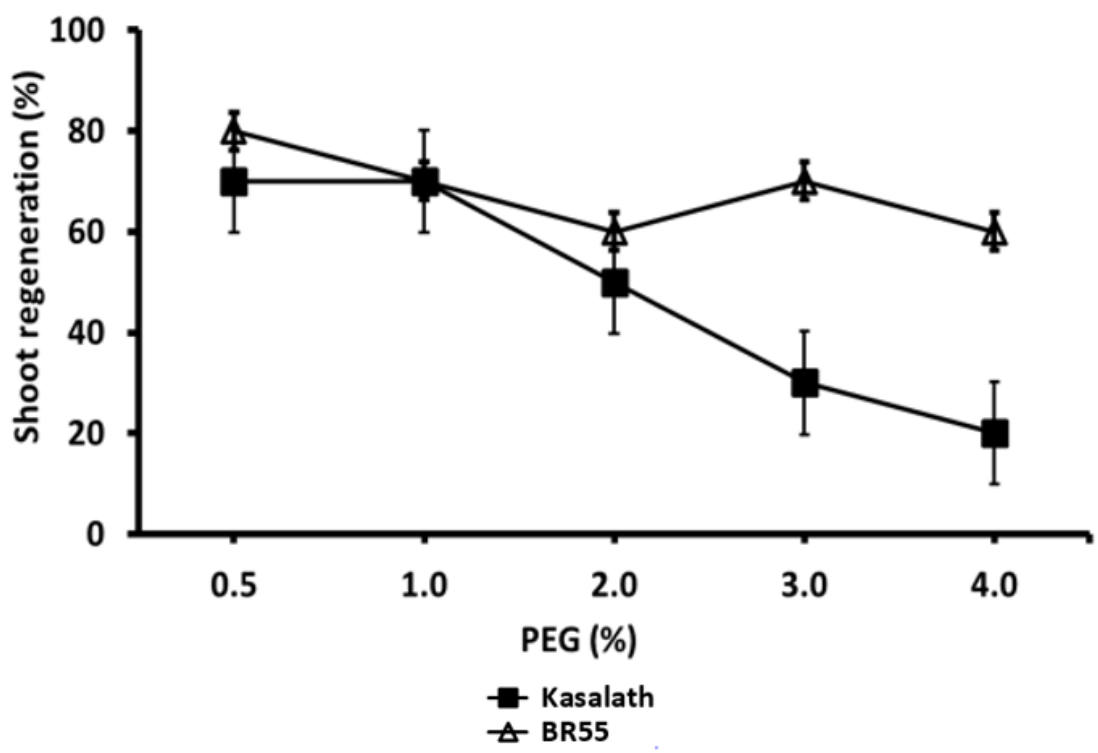

Figure 4. Effect of PEG on Kasalath and BRRI dhan55. The bars are standard errors. 

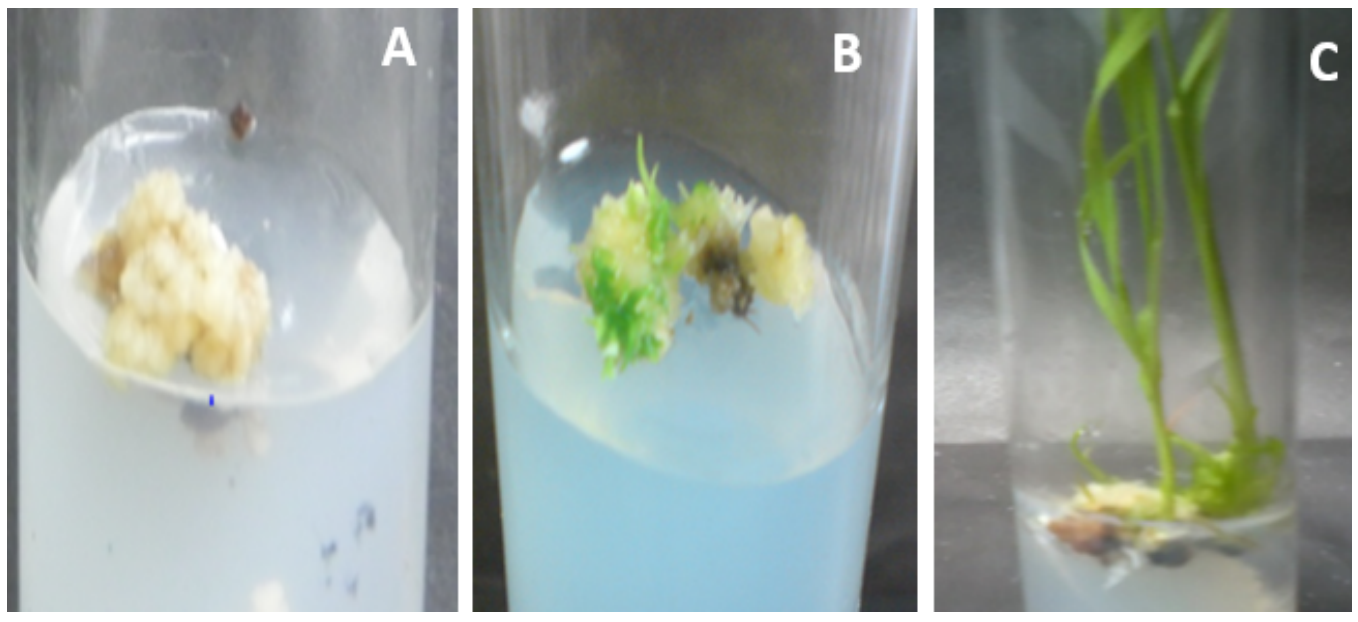

Figure 5. Callus induction and shoot regeneration of rice. A- Callus of Kasalath on callus induction media (MS $+2 \mathrm{mg} / \mathrm{L} 2$, 4-D) after three weeks of inoculation, B- Callus of BRRI dhan55 on shoot regeneration media (MS $+1 \mathrm{mg} / \mathrm{L} \mathrm{NAA}+2 \mathrm{mg} / \mathrm{L} \mathrm{BAP}+1 \mathrm{mg} / \mathrm{L}$ Kinetin), CShoot regeneration of BRRI dhan55 after 5 weeks $(\mathrm{MS}+1 \mathrm{mg} / \mathrm{L} \mathrm{NAA}+2 \mathrm{mg} / \mathrm{L} \mathrm{BAP}+1$ $\mathrm{mg} / \mathrm{L}$ Kinetin $+4 \%$ PEG)

However, BRRI dhan55 had higher shoot regeneration frequency than Kasalath (Figure 4, Figure 5-C). Shoot regeneration frequency for BRRI dhan55 was 70 and $60 \%$ in 3 and $4 \%$ concentration of PEG, respectively. Kasalath were 30 and $20 \%$ in the same concentration of PEG as those of BRRI dhan55. Reduction in regeneration frequency with increased PEG concentration has been observed before.

Wani et al. (2010) showed both reduced callus induction and shoot regeneration of rice cultivars PAU 201 and PR 116 in medium supplement with different concentrations of PEG. Tripathy (2015) also found similar reduction in regeneration frequency with increased concentration of PEG in upland rice. Further, cultivar depended responses with PEG have also been observed. Akte et al. (2016) found that rice cultivar Binadhan-10 performed the best against PEG compared to Binadhan-4, Binadhan-5, Binadhan-6 and Iratom-24. In this study, the difference in PEG tolerance among the two varieties was expected. BRRI dhan55 was developed as a variety that could tolerate medium salt and drought stress (Parvin et al., 2015). Kasalath, on the other hand, is a landrace known for several beneficial traits such as early maturity and can tolerate phosphate deficiency and some levels of drought (Kanamori et al., 2013; Sakai, 2014).

Screening plants for drought tolerance is paramount in sustaining production under drought environments. However, screening under field condition is tedious, resource intensive and time consuming. It is easy to use chemicals to induce artificial drought environment. Therefore, a similar method can be used to select for drought tolerance. PEG (6000) can initiate water stress conditions in nutrient cultures and affect plant cells similarly to those under drought conditions. PEG with high molecular weight (more than 4000) is not 
permeable to plasma cell membrane. It is also not metabolised and not absorbed by plants. However, it decreases water potential in nutrient solution thus stimulate water stress without other negative effects such as toxicity (Wani et al., 2010; Heringer et al., 2013). Thus, the present study further illustrates the benefit that PEG which can be used to screen plantlets for drought stress.

\section{Conclusions}

The present study indicated that the concentrations of auxins and cytokinins were an essential factor of rice callus initiation and shoot regeneration. Polyethylene glycol can be used to induce artificial drought condition in vitro and assess the responses of rice plantlets in early growth stage to drought stress. Thus, it could be an initial step in screening rice cultivars against drought stress.

\section{Acknowledgements}

We acknowledge the Plant Genetic Engineering Laboratory of the Department of Genetic Engineering and Biotechnology, Shahjalal University of Science and Technology for providing facilities to conduct the experiment.

\section{References}

Akte J., Yasmin S., Bhuiyan MJH., Khatun F., Roy J., Goswami K. 2016. In vitro screening of rice genotypes using polyethylene glycol under drought stress. Progressive Agriculture, 27(2): 128-135.

BBS (Bangladesh Bureau of Statistics). 2012. Statistical Year Book of Bangladesh. Ministry of Planning, Government of the People's Republic of Bangladesh. 33-36 pp.

Chakraborty A., Hoque H., Hasan MN., Akter F., Suhani S., Joy ZF., Akther J. 2017. Effect of Different Concentrations of Plant Growth Hormones for in Vitro Regeneration of Rice Varieties BRRI
Dhan 28 and BRRI Dhan 29. International Journal of Sciences: Basic and Applied Research, 33(2): 26-33.

Coenen C., Lomax TL. 1998. The Diageotropica Gene Differentially Affects Auxin and Cytokinin Responses throughout Development in Tomato ${ }^{1}$. 1998. Plant Physiology, 117: 63-72.

FAOSTAT. 2014. Rice production. ttp://www.fao.org/faostat/en/\#data/QC/vis ualize. Retrieved on 01/11/2017.

Haque Z., Chakraborty A., Prodhan SH. 2013. In vitro screening method for drought tolerance evaluation in two rice varieties (BRRI 28 and BRRI 29). International Journal of Scientific \& Engineering Research, 4(6): 339-347.

Heringer AS., Vale EM., Barroso T., SantaCatarina C., Silveira V. 2013. Polyethylene glycol effects on somatic embryogenesis of papaya hybrid UENF/CALIMAN 01 seeds. Theoretical and Experimental Plant Physiology, 25(2), 116-124.

Hussain Z., Khan MH., Bano R., Rashid H., Chaudhry Z. 2010. Protocol optimization for efficient callus induction and regeneration in three Pakistani rice cultivars. Pakistan Journal of Botany, 42(2): 879-887.

IRRI (International Rice Research Institute). 2009. Climate change-ready rice. http://irri.org/ourscience/bettervarieties/cli mate-ready-rice\%23Drought. Retrieved on $02 / 11 / 2017$.

Kacem NS., Delporte F., Muhovski Y., Djekoun A., Watillon B. 2017. In vitro screening of durum wheat against water-stress mediated through polyethylene glycol. Journal of Genetic Engineering and Biotechnology, 15: 239-247.

Kanamori H., Fujisawa M., Katagiri S., Oono Y., Fujisawa H., Karasawa W., Kurita K., Sasaki H., Mori S., Hamada M., Mukai Y., Yazawa T., Mizuno H., Namiki N., 
Sasaki T., Katayose Y., Matsumoto T., Wu J. 2013. A BAC physical map of aus rice cultivar 'Kasalath', and the mapbased genomic sequence of 'Kasalath' chromosome 1. The Plant Journal, 76: 699-708.

Kulkarni M., Deshpande U. 2007. In Vitro screening of tomato genotypes for drought resistance using polyethylene glycol. African Journal of Biotechnology, 6(6): 691-696.

Mahmood R., Legates DR., Meo M. 2004. The Role of Soil Water Availability in Potential Rainfed Rice Productivity in Bangladesh: Applications of the CERESRice Model. Applied Geography, 24: 139159.

Meher, Shivakrishna P., Reddy KA., Rao DM. 2017. Effect of PEG-6000 imposed drought stress on RNA content, relative water content (RWC), and chlorophyll content in peanut leaves and roots. Saudi Journal of Biological Sciences, 25: 285-289.

Mohd Din ARJ., Ahmad FI., Wagiran A., Samad AA., Rahmat Z., Sarmidi MR. 2016. Improvement of efficient in vitro regeneration potential of mature callus induced from Malaysian upland rice seed (Oryza sativa cv. Panderas). Saudi Journal of Biological Sciences, 23: S69S77.

Pandey S., Bhandari H. 2006. Drought perpetuates poverty. Rice Today, 5:37.

Parvin S., Biswas S., Razzaque S., Haque T., Elias SM., Tammi RS., Seraj ZI. 2015. Salinity and drought tolerance conferred by in planta transformation of SNAC1 transcription factor into a high-yielding rice variety of Bangladesh. Acta Physiology Plant, 37:68.

Pawar B., Kale P., Bahurupe J., Jadhav A., Kale A., Pawar S. 2015. Proline and Glutamine Improve in vitro Callus Induction and
Subsequent Shooting in Rice. Rice Science, 22(6): 283-289.

Piwowarczyk B., Kamińska I., Rybiński W. 2014. Influence of PEG Generated Osmotic Stress on Shoot Regeneration and Some Biochemical Parameters in Lathyrus Culture. Czech Journal of Genetics and Plant Breeding, 50: 77-83.

Phung TH., Jung H., Park JH., Kim JG., Back K., Jung S. 2011. Porphyrin Biosynthesis Control under Water Stress: Sustained Porphyrin Status Correlates with Drought Tolerance in Transgenic Rice. Plant Physiology, 157: 1746-1764.

Sabesan T., Saravanan K. 2016. In Vitro Screening of Indica Rice Genotypes for Drought Tolerance Using Polyethylene Glycol. International Journal of Advances in Agricultural and Environmental Engineering, 3(2): 2349-1523.

Sakai H. 2014. Construction of Pseudomolecule Sequences of the aus Rice Cultivar Kasalath for Comparative Genomics of Asian Cultivated Rice. DNA Research, 21: 397-405.

Shahsavari E., Maheran AA., Siti Nor Akmar A., Hanafi MM. 2010. The effect of plant growth regulators on optimization of tissue culture system in Malaysian upland rice. African Journal of Biotechnology, 9(14): 2089-2094.

Swamy BPM., Kumar A. 2012. Sustainable rice yield in water-short drought-prone environments: Conventional and molecular approaches. Irrigation Systems and Practices In: Challenging Environments, (Ed.) Dr. Teang Shui Lee, ISBN: 978-953-51-0420-9, In Tech.

Tripathy SK. 2015. In Vitro Screening of Callus Cultures and Regenerants for Drought Tolerance in Upland Rice. Research Journal of Biotechnology, 10(6): 23-28.

Upadhyaya G., Sen M., Roy A. 2015. In vitro callus induction and plant regeneration of rice (Oryza sativa L.) var. 'Sita', 'Rupali' 
and 'Swarna Masuri'. Asian Journal of Plant Science and Research, 5(5): 24-27.

Wani SH., Sofi PA., Gosal SS., Singh NB. 2010. In vitro screening of rice (Oryza sativa $\mathrm{L}$ ) callus for drought tolerance. Communications in Biometry and Crop Science, 5(2): 108-115.
Wani SH., Sanghera GS., Gosal SS. 2011. An efficient and reproducible method for regeneration of whole plants from mature seeds of a high yielding Indica rice (Oryza sativa L.) variety PAU 201. Net Biotechnology, 4(28): 418-422. 